\title{
Novel Ion Extraction Method for Imaging Mass Spectrometry
}

\author{
Jun Aoki, ${ }^{1,3,} *$ Hisanao Hazama, ${ }^{2,3}$ and Michisato Toyoda ${ }^{1,3}$ \\ ${ }^{1}$ Graduate School of Science, Osaka University, Toyonaka, OSAKA, JAPAN \\ ${ }^{2}$ Graduate School of Enginnering, Osaka University, Suita, OSAKA, JAPAN \\ ${ }^{3}$ Japan Science and Technology Agency, CREST, Chiyoda-ku, TOKYO, JAPAN
}

\begin{abstract}
We have developed a new ion extraction method for a stigmatic matrix-assisted laser desorption/ionization (MALDI) imaging time-of-flight (TOF) mass spectrometer. The conventional delayed extraction method is unsuitable for a stigmatic imaging TOF mass spectrometer because the ions disperse until extraction and the ion distribution is not preserved for imaging. In the proposed method, temporal and spatial focusing can be simultaneously achieved, enabling a high-fidelity image to be obtained with a high mass resolution. This method is expected to be applicable to cellular-level observations by imaging mass spectrometry.
\end{abstract}

(Received January 11, 2011; Accepted March 15, 2011)

\section{Introduction}

Analytical techniques for measuring two-dimensional distributions of molecules such as proteins and drugs on a cellular level are desired in many fields including pathology and pharmacology. Recently, scanning imaging mass spectrometry (IMS) with matrix-assisted laser desorption/ionization (MALDI) $)^{1)}$ has been intensively used for biomolecular analysis..2), 3) IMS analysis is usually performed by raster scanning a focused laser beam over the imaging area and obtaining mass spectra of ions generated at each exposure point. ${ }^{3)}$ The resultant data set consists of several mass spectra and their positional information (i.e., the $x-y$ coordinates of each exposure point). An image showing the spatial distribution for a specific $m / z$ is computed by extracting the peak intensity as a function of position from the data set. In scanning mode, the lateral resolution is roughly regulated by the beam size. In practical applications, the smallest beam size for MALDI is several tens of micrometers, which is too large for cellular level observations.

To overcome this limitation, stigmatic MALDI imaging mass spectrometry has been developed, which is based on time-of-flight (TOF) mass spectrometry. ${ }^{4)}$ In this stigmatic MALDI imaging mass spectrometry, the spatial distributions of ions at the sample surface are maintained and projected onto a position-sensitive ion detector. This enables a spatial resolution of the order of micrometers to be achieved irrespective of the laser spot diameter. Scanning imaging mass spectrometers were developed by using the existing technology of MALDI-TOF mass spectrometers. In contrast, new techniques are required to develop a stigmatic MALDITOF imaging mass spectrometer. Several new tech-

* Correspondence to: Jun AoKI, Graduate School of Science, Osaka University, 1-1 Machikaneyama, Toyonaka, OSAKA 565-0043, JAPAN, e-mail: ajunmail@mass.phys. sci.osaka-u.ac.jp niques have been developed for stigmatic imaging mass spectrometers; for example, conservation of the image of ions during ion extraction from the sample surface $^{4)}$ and during flight in the mass analyzer ${ }^{4-6)}$ and simultaneous detection of the arrival position and the flight time of an ion.5), 7) As a result of these developments, several stigmatic MALDI-TOF imaging mass spectrometers have been constructed. ${ }^{4), 6)}$

At this stage, the mass resolutions obtained from these apparatuses are too low for biomolecular analysis. The delayed extraction technique is employed in conventional MALDI-TOF mass spectrometers to improve their mass resolutions, ${ }^{8)-10)}$ though the delayed extraction technique cannot be used in stigmatic imaging mass spectrometers because the ion image is disturbed during the delay time. Typically, TOF mass spectrometer with no delayed extraction have a mass resolution of only $m / \Delta m<100$, which is practically useless. We have developed a new extraction method for stigmatic imaging TOF mass spectrometers that achieves both high-fidelity imaging and a high mass resolution. This paper describes the concept of this new extraction method and presents experimental results obtained using it.

\section{Ion Extraction Method}

\subsection{Conventional delayed extraction method}

The initial ion velocities in MALDI of $500-1,000 \mathrm{~m} / \mathrm{s}$ with a dispersion of several hundreds of meters per second have been achieved. ${ }^{11), 12)}$ To minimize the effect of the dispersion in the initial velocity distribution on the mass resolution, the delayed extraction method is widely used to compensate the dispersion in the initial ion velocity. Although the delayed extraction method is essential in MALDI-TOF mass spectrometers, it cannot be applied to stigmatic imaging mass spectrometers. In stigmatic imaging mass spectrometers, ions diffuse in a plane perpendicular to the flight axis during the delay time prior to extraction; thus, the ion distribution is not preserved so that it 


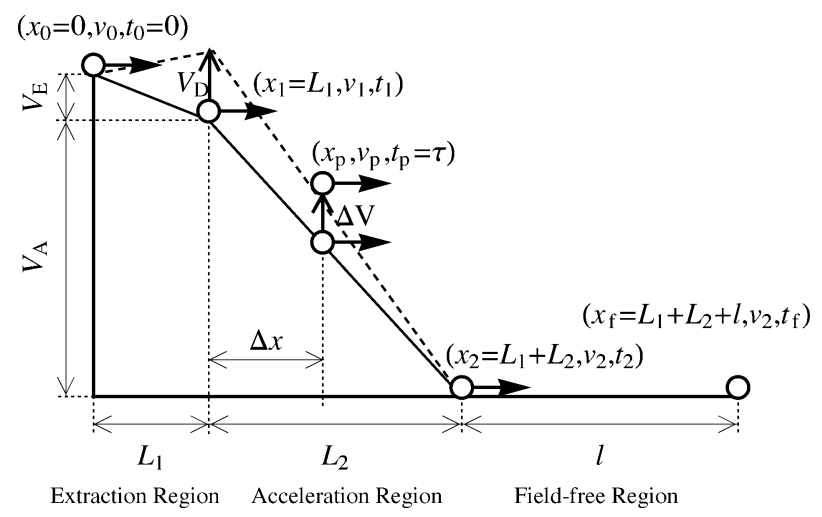

Fig. 1. Schematic of post extraction differential acceleration operation. Solid line represents an initial potential, and dashed line represents a raised potential after a delay time of $\tau$.

cannot be observed as an image. In stigmatic imaging mass spectrometers, ions are extracted immediately without employing any method for compensating the velocity dispersion. In this approach, the ion image is conserved, but the mass resolution is very low.

\subsection{Post-extraction differential acceleration}

We have developed a new method for compensating the initial velocity dispersion that does not reduce the image quality. Here, we explain the operation procedure of this new ion extraction method, which we term "post-extraction differential acceleration (PEDA)." Figure 1 shows the schematic of this extraction method. In the delayed extraction method, the image quality deteriorates during the delay time before the extraction operation. In the case of PEDA, an ion generated at $x=0$ with an initial velocity of $v_{0}$ is extracted immediately after ionization by the electric field in the extraction region with a voltage of $V_{\mathrm{E}}$. When the ion passes through the extraction electrode at $x=L_{1}$ after a flight time of $t_{1}$, the ion velocity is $v=v_{1}$. Here, $v_{1}$ is represented as follows with the given conditions.

$$
v_{1}=\sqrt{\frac{2}{m}\left(\frac{1}{2} m v_{0}^{2}+e V_{\mathrm{E}}\right)}
$$

Here, $m$ is the ion mass and $e$ is the elementary charge. $t_{1}$ satisfies the following equation.

$$
v_{0} t_{1}+\frac{1}{2} \frac{e}{m} \frac{V_{\mathrm{E}}}{L_{1}} t_{1}^{2}=L_{1}
$$

Solving Eq. (2) gives:

$$
t_{1}=\frac{m v_{0} L_{1}}{e V_{\mathrm{E}}}\left(-1+\sqrt{1+e V_{\mathrm{E}} / \frac{1}{2} m v_{0}^{2}}\right) .
$$

The ion is further accelerated in the acceleration region by an electric field of $V_{\mathrm{A}} / L_{2}$. At $t=\tau$, the voltage applied to the extraction electrode is increased by $V_{\mathrm{D}}$, which increases the electric field in the acceleration region from $V_{\mathrm{A}} / L_{2}$ to $\left(V_{\mathrm{A}}+V_{\mathrm{D}}\right) / L_{2}$. At that time, the ion is located at $x=x_{\mathrm{p}}=x_{1}+\Delta x$ with a velocity of $v=v_{\mathrm{p}}$. $\Delta x$ is represented as

$$
\Delta x=v_{1}\left(\tau-t_{1}\right)+\frac{1}{2} \frac{e}{m} \frac{V_{\mathrm{A}}}{L_{2}}\left(\tau-t_{1}\right)^{2} .
$$

The voltage increment at $x=x_{\mathrm{p}}$ is represented as

$$
\Delta V=V_{\mathrm{D}}\left(1-\frac{\Delta x}{L_{2}}\right) .
$$

Thus, the incremental energy of the ion is proportional to the distance from the acceleration electrode. By using the energy conservation law, $v_{\mathrm{p}}$ is derived as

$$
v_{\mathrm{p}}=\sqrt{\frac{2}{m}\left(\frac{1}{2} m v_{0}^{2}+e\left(V_{\mathrm{E}}+V_{\mathrm{A}} \frac{\Delta x}{L_{2}}\right)\right)} .
$$

The ion is then accelerated in the modified field until reaching $x=L_{1}+L_{2}$ with a velocity of $v_{2}$ at $t=t_{2}$. $v_{2}$ is derived as

$$
v_{2}=\sqrt{\frac{2}{m}\left(\frac{1}{2} m v_{0}^{2}+e\left(V_{\mathrm{E}}+V_{\mathrm{A}}+\Delta V\right)\right)} .
$$

$t_{2}$ satisfies the following equation

$$
v_{\mathrm{p}}\left(t_{2}-\tau\right)+\frac{1}{2} \frac{e}{m} \frac{V_{\mathrm{A}}+V_{\mathrm{D}}}{L_{2}}\left(t_{2}-\tau\right)^{2}=L_{2}-\Delta x,
$$

and is derived as

$$
\begin{aligned}
t_{2}= & \tau+\frac{m v_{\mathrm{p}} L_{2}}{e\left(V_{\mathrm{A}}+V_{\mathrm{D}}\right)} \\
& \times\left(\sqrt{1+e\left(V_{\mathrm{A}}+V_{\mathrm{D}}\right)\left(1-\frac{\Delta x}{L_{2}}\right) /\left(\frac{1}{2} m v_{\mathrm{p}}^{2}\right)-1}\right) .
\end{aligned}
$$

By increasing the potential in this manner, the ion gains an additional kinetic energy that depends on its position in the axial direction at $t=\tau$. Because the axial position of the ion depends on its initial velocity, as expressed by Eq. (4), an ion with a high initial velocity located at the front will gain less energy than an ion with a low initial velocity located at the back. After acceleration, the ion passes through the field-free region with length $l$ to a detector in a time of $l / v_{2}$. Thus, the total time of flight of the ion is

$$
t_{\mathrm{f}}=t_{2}+\frac{l}{v_{2}} .
$$

For time focusing to obtain a high mass resolution, $t_{\mathrm{f}}$ should be constant for any $v_{0}$. In actual experiments, since the dimensions of the apparatus are fixed and the voltages that can be applied to the electrodes are limited, the delay time $\tau$ is the dominant variable parameter. Figure 2 shows the time variance of $t_{\mathrm{f}}$ plotted as a function of the initial velocity $v_{0}$ and the delay time $\tau$. Other parameters are set to their experimental values, namely $V_{\mathrm{A}}=4,900 \mathrm{~V}, V_{\mathrm{E}}=100 \mathrm{~V}, V_{\mathrm{D}}=500 \mathrm{~V}, L_{1}=3.5$ $\mathrm{mm}, L_{2}=20 \mathrm{~mm}, l=790 \mathrm{~mm}$, and $m / z$ 373.25. Time focusing is achieved when the time variance becomes flat to the initial velocity axis. We assumed that the initial ion velocity is distributed as $v=800 \pm 500$ $\left.\mathrm{m} / \mathrm{s}^{11)}, 12\right)$; this condition is generally satisfied when the time variance is less than 4 ns for $\tau=1.18 \mu$ s (see Fig. 2(b)). The calculated value of $\tau$ agrees well with the experimental results given below. This focusing condition is changed due to $m / z$ as similar to the conventional delayed extraction. The practical range is estimated as approximately $\pm 20 \%$ of optimum $\mathrm{m} / \mathrm{z}$.

\section{Experimental Apparatus}

Figure 3 shows the apparatus used in the present 


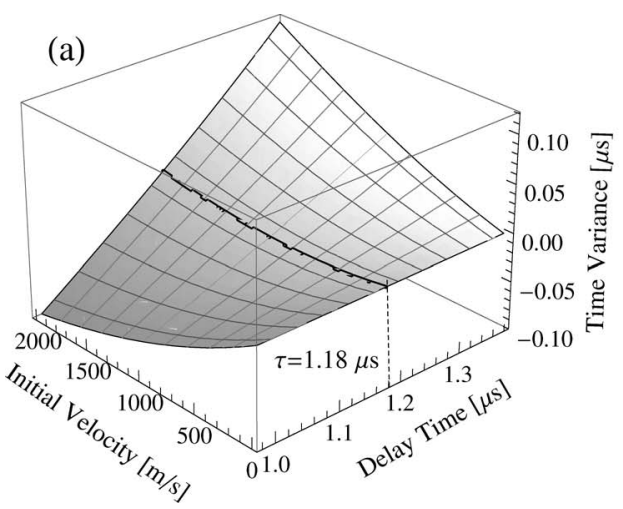

(b)

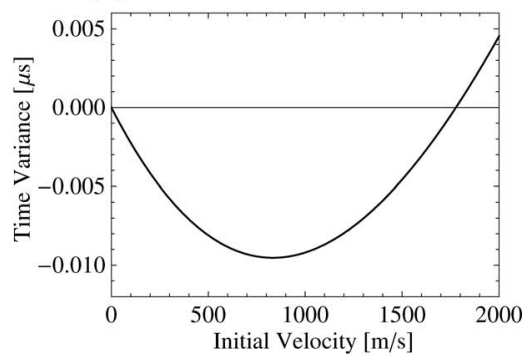

Fig. 2. (a) Analytical plot of variance of flight time using PEDA as functions of initial velocity and delay time $\tau$ and (b) cross-sectional profile of $\tau=1.18 \mu \mathrm{s}$.

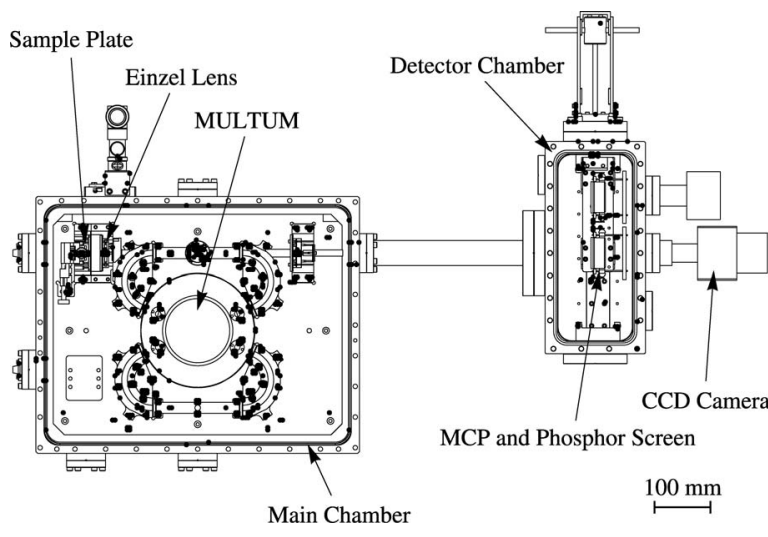

Fig. 3. Experimental apparatus.

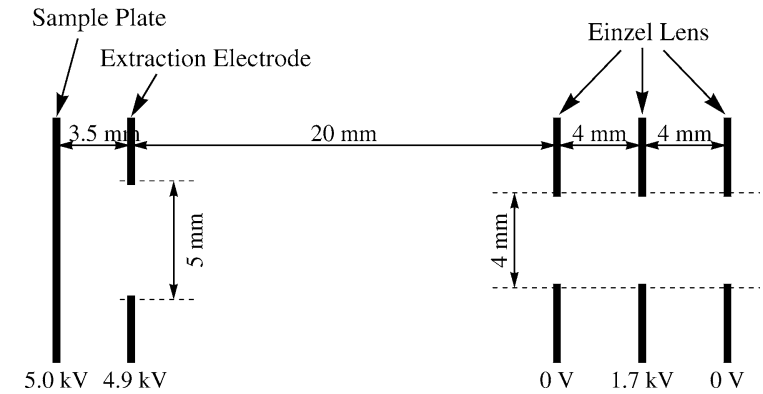

Fig. 4. Schematic of the ion source showing dimensions and typical electrode voltages.

experiments. It consists of a MALDI ion source, a multi-turn flight path, ${ }^{13), 14)}$ and detector made up of a microchannel plate (MCP) and a phosphor screen (F2223-27P MOD, Hamamatsu Photonics, Hamamatsu, Japan). Figure 4 shows a detailed diagram of the ion source. The third harmonic of a Nd: YAG laser (GAIAII 50T, Rayture Systems, Tokyo, Japan) was introduced into the MALDI ion source and focused on the sample plate to a diameter of about $800 \mu \mathrm{m}$. The ions generated from the sample on the sample plate are extracted by the electric field applied between the sample plate and the extraction electrode, which are separated by $3.5 \mathrm{~mm}$. The extraction electrode is a disk with a 5-mm-diameter aperture from which ions are extracted. The extracted ions are accelerated in the 20mm-long acceleration region by the electric field between the extraction electrode and the acceleration

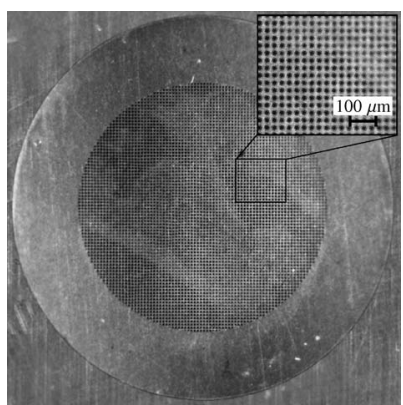

Fig. 5. An optical micrograph of the evaluation sample (a dried droplet of crystal violet dye covered by a fine metal grid).

electrode, which was also utilized as the first electrode of the Einzel lens. The Einzel lens consists of three disk electrodes with 4-mm-diameter apertures that have separations of $4 \mathrm{~mm}$. The extracted ions are focused by the Einzel lens onto the MCP assembly. The ion distribution is observed as a luminescence image on the phosphor screen by using a CCD camera (ORCA-ER1394, Hamamatsu Photonics, Hamamatsu, Japan). The MCP output signal is measured at the same time to obtain the TOF spectrum. Although a multi-turn mass spectrometer (MULTUM ${ }^{13), 14)}$ ) is installed in the flight path, it was not used in this experiment.

\section{Results and Discussion}

\subsection{Experimental conditions}

A dried droplet of a saturated aqueous solution of crystal violet dye covered by a fine metal grid with a pitch of $25.4 \mu \mathrm{m}$ (G1000HS; 1,000 lines/inch, Gilder Grids, Grantham, UK), a $6-\mu \mathrm{m}$ bar, and a $19.4-\mu \mathrm{m}$ hole, was used to evaluate the imaging performance. The ion distribution is observed as a fine grid of squares. Figure 5 shows an optical micrograph of the metal grid sample. The sample was ionized by an $800-\mu \mathrm{m}-$ diameter laser beam. Voltages of $5.0 \mathrm{kV}$ and $4.9 \mathrm{kV}$ were applied to the sample plate and the extraction electrode, respectively. When the PEDA method was used, the voltage applied to the extraction electrode was switched to $5.4 \mathrm{kV}$ at a delay time of $1.15 \mu$ s after the laser pulse. This delay time was experimentally determined by adjusting it until the TOF peak width was minimized. The voltage applied to the Einzel lens was $1.74 \mathrm{kV}$ for normal extraction and $1.80 \mathrm{kV}$ in the 


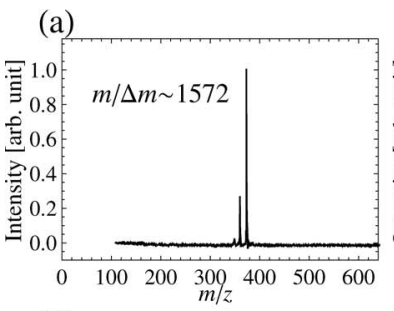

(d)

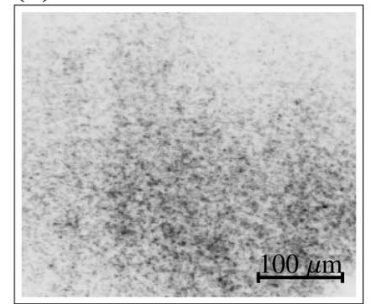

(b)

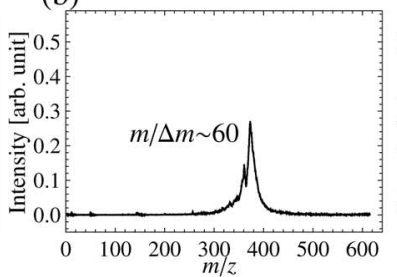

(e)

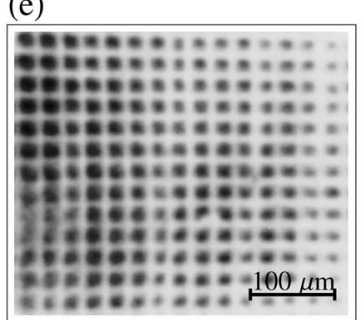

(c)

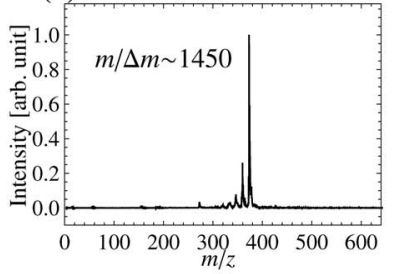

(f)

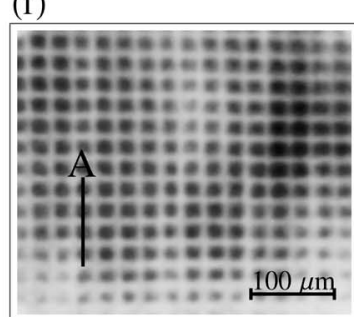

Fig. 6. Experimental TOF spectra for (a) the conventional delayed extraction method, (b) the normal extraction method, and (c) PEDA method; (d-f) corresponding ion images.

PEDA method.

\subsection{Time focusing and image focusing}

Figure 6 shows the obtained TOF spectra and ion images. Figures 6(a), (b), and (c) show TOF spectra obtained using conventional delayed extraction, normal extraction, and the PEDA method, respectively. The peak width is clearly much narrower for PEDA than for the normal extraction method, being similar to that obtained by conventional delayed extraction. Mass resolutions of $m / \Delta m \approx 1,572$ for delayed extraction, $m / \Delta m \approx 60$ for normal extraction, and $m / \Delta m \approx$ 1,450 for PEDA were obtained for the peak at $m / z$ 373.25. Figure 7 shows the experimentally observed peak width variance as a function of the delay time $\tau$ for PEDA. The minimum peak width occurs at $\tau=1.10$ to 1.15. Figure 7 compares the experimental and numerical calculation results. The flight time variance obtained by numerical calculations is a minimum at $\tau=$ 1.18. The two results have a shift of about $0.05 \mu \mathrm{s}$ in the delay time. This shift is considered to be due to the time lag in the circuit used in this experiment.

Figures 6(d), (e), and (f) show ion images obtained using conventional delayed extraction, normal extraction, and PEDA. For the delayed extraction method, the ions are diffused and do not form a focused image, as shown in Fig. 6(d). Figure 8 shows the intensity line profile along line A in Fig. 6(f). To evaluate the image quality of Figs. 6(e) and (f), we calculated their spatial resolutions. The spatial resolution is defined as the distance along the profile for which the intensity increases from $20 \%$ to $80 \%$ of the maximum value. ${ }^{15}$ The spatial resolutions of the peaks were in the range $4.0 \mu \mathrm{m}$ to $5.5 \mu \mathrm{m}$ along line A. Figures 9 (a) and (b) show frequency distributions for the spatial resolutions of all peaks in the images for normal extraction and PEDA, respectively. We fitted a Gaussian distribution of

$$
f(x)=A \exp \left(-\frac{(x-\mu)^{2}}{2 \sigma^{2}}\right)
$$

to evaluate the distribution shapes. Normal extraction and PEDA had means of $\mu=4.9$ and $\mu=4.7$ and variances of $\sigma^{2}=0.48$ and $\sigma^{2}=0.43$, respectively. The two

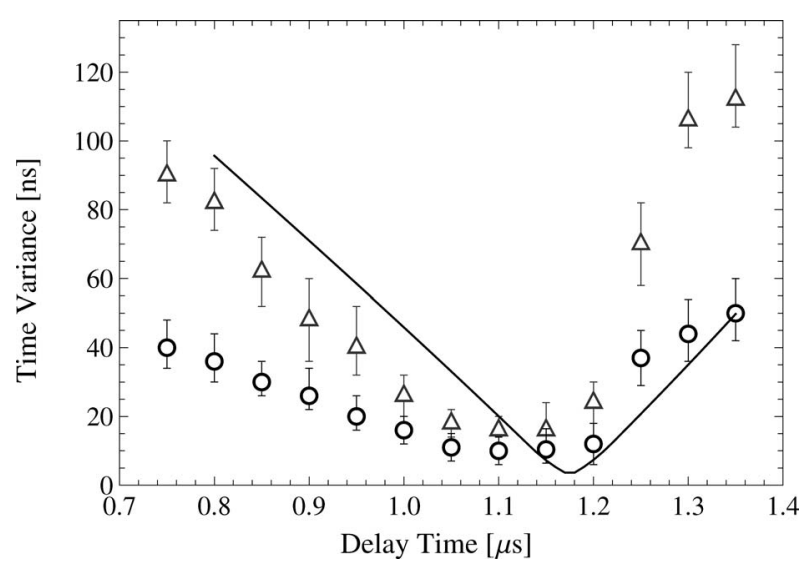

Fig. 7. Variance of flight time plotted as a function of delay time $\tau$. Solid line represents calculated time variance of ions with initial velocity of 800 $\pm 500 \mathrm{~m} / \mathrm{s}$; circles and triangles represent experimental peak widths at half height and $10 \%$ height, respectively.

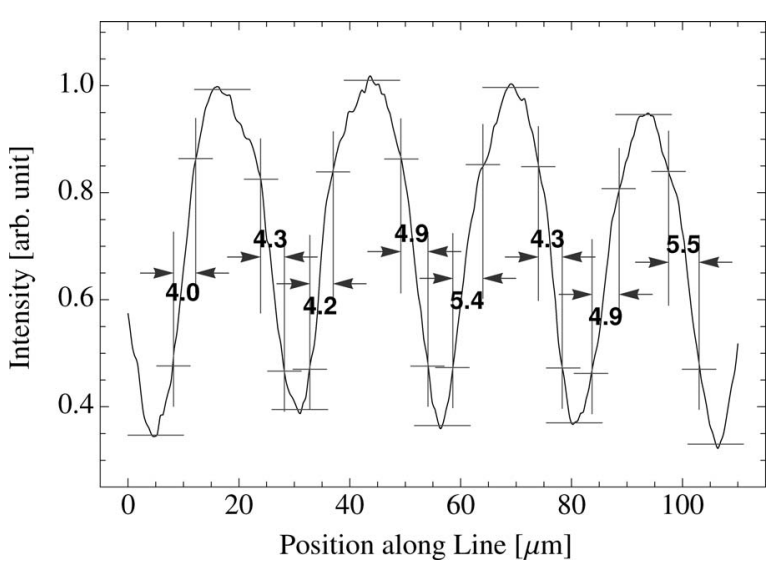

Fig. 8. Line profile of grid image along line A in Fig. 6(d). Spatial resolution is evaluated for each peak. 


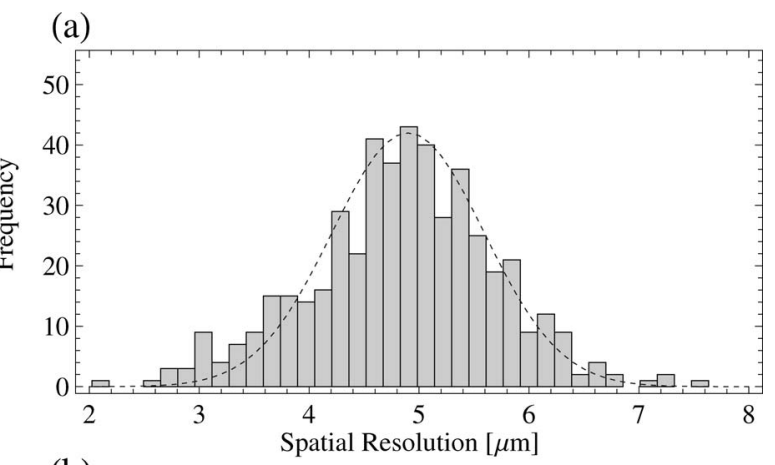

(b)

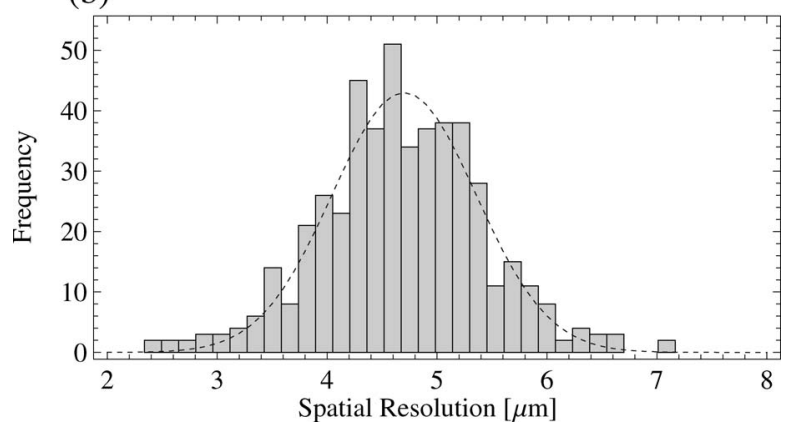

Fig. 9. Frequency distributions of spatial resolutions for (a) normal extraction method and (b) PEDA method.

methods give similar frequency distributions. Therefore, the spatial resolution is maintained by the PEDA method.

\section{Conclusion}

We have developed a new ion extraction method for MALDI imaging TOF mass spectrometers. In this new method, a similar time focusing is achieved as that of the conventional delayed extraction method and the ion distribution image quality is conserved. In an evaluation experiment of the new method, we achieved a mass resolution of $m / \Delta m \approx 1,450$ with a flight length of $790 \mathrm{~mm}$ and the spatial resolution of the simultaneously obtained image is equal to that of the normal extraction, whereas the mass resolution using normal extraction is $m / \Delta m \approx 60$. The experimental parameter of the delay time $\tau$ agreed well with the analytical result. Using this method, the mass resolution of a stigmatic MALDI imaging TOF mass spectrometer can be improved without reducing the image quality.

\section{Acknowledgments}

This work was supported by the Japan Science and Technology Agency, CREST.

\section{References}

1) F. Hillenkamp, M. Karas, R. C. Beavis, and R. Nitsche, Anal. Chem., 63, 1193 (1991).

2) R. M. Caprioli, T. B. Farmer, and J. Gile, Anal. Chem., 69, 4751 (1997).

3) L. A. McDonnell and R. M. A. Heeren, Mass Spectrom. Rev., 26, 606 (2007).

4) S. L. Luxembourg, T. H. Mize, L. A. McDonnell, and R. M. A. Heeren, Anal. Chem., 76, 5339 (2004).

5) B. W. Schueler, Microsc. Microanal. Microstruct., 3, 119 (1992).

6) H. Hazama, J. Aoki, H. Nagao, R. Suzuki, T. Tashima, K. Fujii, K. Masuda, K. Awazu, M. Toyoda, and Y. Naito, Appl. Surf. Sci., 255, 1257 (2008).

7) O. Jagutzki, V. Mergel, K. Ullmann-Pfleger, L. Spielberger, U. Meyer, R. Doerner, and H. W. Schmidt-Boecking, Proc. SPIE, 3438, 322 (1998).

8) W. C. Wiley and I. H. McLaren, Rev. Sci. Instrum., 26, 1150 (1955).

9) S. M. Colby, T. B. King, J. P. Reilly, and D. M. Lubman, Rapid Commun. Mass Spectrom., 8, 865 (1994).

10) R. S. Brown and J. J. Lennon, Anal. Chem., 67, 1998 (1995).

11) R. C. Beavis and B. T. Chait, Chem. Phys. Lett., 181, 479 (1991).

12) A. A. Puretzky, D. B. Geohegan, G. B. Hurst, and M. V. Buchanan, Phys. Rev. Lett., 83, 444 (1999).

13) M. Toyoda, D. Okumura, M. Ishihara, and I. Katakuse, J. Mass Spectrom., 38, 1125 (2003).

14) M. Toyoda, Eur. J. Mass Spectrom., 16, 397 (2010).

15) T. L. Colliver, C. L. Brummel, M. L. Pacholski, F. D. Swanek, A. G. Ewing, and N. Winograd, Anal. Chem., 69, 2225 (1997).

Keywords: Stigmatic imaging mass spectrometry, Ion extraction method 